\title{
Adapting the Neural Encoder-Decoder Framework from Single to Multi-Document Summarization
}

\author{
Logan Lebanoff, Kaiqiang Song and Fei Liu \\ Department of Computer Science \\ University of Central Florida, Orlando, FL 32816, USA \\ \{loganlebanoff, kqsong\}@knights.ucf.edu feiliu@cs.ucf.edu
}

\begin{abstract}
Generating a text abstract from a set of documents remains a challenging task. The neural encoder-decoder framework has recently been exploited to summarize single documents, but its success can in part be attributed to the availability of large parallel data automatically acquired from the Web. In contrast, parallel data for multi-document summarization are scarce and costly to obtain. There is a pressing need to adapt an encoder-decoder model trained on single-document summarization data to work with multiple-document input. In this paper, we present an initial investigation into a novel adaptation method. It exploits the maximal marginal relevance method to select representative sentences from multi-document input, and leverages an abstractive encoder-decoder model to fuse disparate sentences to an abstractive summary. The adaptation method is robust and itself requires no training data. Our system compares favorably to state-of-the-art extractive and abstractive approaches judged by automatic metrics and human assessors.
\end{abstract}

\section{Introduction}

Neural abstractive summarization has primarily focused on summarizing short texts written by single authors. For example, sentence summarization seeks to reduce the first sentence of a news article to a title-like summary (Rush et al., 2015; Nallapati et al., 2016; Takase et al., 2016; Song et al., 2018); single-document summarization (SDS) focuses on condensing a news article to a handful of bullet points (Paulus et al., 2017; See et al., 2017). These summarization studies are empowered by large parallel datasets automatically harvested from online news outlets, including Gigaword (Rush et al., 2015), CNN/Daily Mail (Hermann et al., 2015), NYT (Sandhaus, 2008), and Newsroom (Grusky et al., 2018).

To date, multi-document summarization (MDS) has not yet fully benefited from the development

\begin{tabular}{|c|c|c|c|}
\hline DATASET & SOURCE & SUMMARY & \#PAIRS \\
\hline $\begin{array}{l}\text { Gigaword } \\
\text { (Rush et al., 2015) }\end{array}$ & $\begin{array}{l}\text { the first sentence } \\
\text { of a news article }\end{array}$ & $\begin{array}{l}8.3 \text { words } \\
\text { title-like }\end{array}$ & 4 Million \\
\hline $\begin{array}{l}\text { CNN/Daily Mail } \\
\text { (Hermann et al., 2015) }\end{array}$ & a news article & $\begin{array}{l}56 \text { words } \\
\text { multi-sent }\end{array}$ & $312 \mathrm{~K}$ \\
\hline $\begin{array}{l}\text { TAC (08-11) } \\
\text { (Dang et al., 2008) }\end{array}$ & $\begin{array}{l}10 \text { news articles } \\
\text { related to a topic }\end{array}$ & $\begin{array}{l}100 \text { words } \\
\text { multi-sent }\end{array}$ & 728 \\
\hline $\begin{array}{l}\text { DUC (03-04) } \\
\text { (Over and Yen, 2004) }\end{array}$ & $\begin{array}{l}10 \text { news articles } \\
\text { related to a topic }\end{array}$ & $\begin{array}{l}100 \text { words } \\
\text { multi-sent }\end{array}$ & 320 \\
\hline
\end{tabular}

Table 1: A comparison of datasets available for sent. summarization (Gigaword), single-doc (CNN/DM) and multi-doc summarization (DUC/TAC). The labelled data for multi-doc summarization are much less.

of neural encoder-decoder models. MDS seeks to condense a set of documents likely written by multiple authors to a short and informative summary. It has practical applications, such as summarizing product reviews (Gerani et al., 2014), student responses to post-class questionnaires (Luo and Litman, 2015; Luo et al., 2016), and sets of news articles discussing certain topics (Hong et al., 2014). State-of-the-art MDS systems are mostly extractive (Nenkova and McKeown, 2011). Despite their promising results, such systems cannot perform text abstraction, e.g., paraphrasing, generalization, and sentence fusion (Jing and McKeown, 1999). Further, annotated MDS datasets are often scarce, containing only hundreds of training pairs (see Table 1). The cost to create ground-truth summaries from multiple-document inputs can be prohibitive. The MDS datasets are thus too small to be used to train neural encoder-decoder models with millions of parameters without overfitting.

A promising route to generating an abstractive summary from a multi-document input is to apply a neural encoder-decoder model trained for singledocument summarization to a "mega-document" created by concatenating all documents in the set at test time. Nonetheless, such a model may not scale well for two reasons. First, identifying important text pieces from a mega-document can be challenging for the encoder-decoder model, which is trained on single-document summarization data 
where the summary-worthy content is often contained in the first few sentences of an article. This is not the case for a mega-document. Second, redundant text pieces in a mega-document can be repeatedly used for summary generation under the current framework. The attention mechanism of an encoder-decoder model (Bahdanau et al., 2014) is position-based and lacks an awareness of semantics. If a text piece has been attended to during summary generation, it is unlikely to be used again. However, the attention value assigned to a similar text piece in a different position is not affected. The same content can thus be repeatedly used for summary generation. These issues may be alleviated by improving the encoder-decoder architecture and its attention mechanism (Cheng and Lapata, 2016; Tan et al., 2017). However, in these cases the model has to be re-trained on large-scale MDS datasets that are not available at the current stage. There is thus an increasing need for a lightweight adaptation of an encoder-decoder model trained on SDS datasets to work with multidocument inputs at test time.

In this paper, we present a novel adaptation method, named PG-MMR, to generate abstracts from multi-document inputs. The method is robust and requires no MDS training data. It combines a recent neural encoder-decoder model (PG for Pointer-Generator networks; See et al., 2017) that generates abstractive summaries from singledocument inputs with a strong extractive summarization algorithm (MMR for Maximal Marginal Relevance; Carbonell and Goldstein, 1998) that identifies important source sentences from multidocument inputs. The PG-MMR algorithm iteratively performs the following. It identifies a handful of the most important sentences from the megadocument. The attention weights of the PG model are directly modified to focus on these important sentences when generating a summary sentence. Next, the system re-identifies a number of important sentences, but the likelihood of choosing certain sentences is reduced based on their similarity to the partially-generated summary, thereby reducing redundancy. Our research contributions include the following:

- we present an investigation into a novel adaptation method of the encoder-decoder framework from single- to multi-document summarization. To the best of our knowledge, this is the first attempt to couple the maximal marginal relevance algorithm with pointer-generator networks for multi-document summarization;
- we demonstrate the effectiveness of the proposed method through extensive experiments on standard MDS datasets. Our system compares favorably to state-of-the-art extractive and abstractive summarization systems measured by both automatic metrics and human judgments.

\section{Related Work}

Popular methods for multi-document summarization have been extractive. Important sentences are extracted from a set of source documents and optionally compressed to form a summary (Daume III and Marcu, 2002; Zajic et al., 2007; Gillick and Favre, 2009; Galanis and Androutsopoulos, 2010; Berg-Kirkpatrick et al., 2011; Li et al., 2013; Thadani and McKeown, 2013; Wang et al., 2013; Yogatama et al., 2015; Filippova et al., 2015; Durrett et al., 2016). In recent years neural networks have been exploited to learn word/sentence representations for single- and multi-document summarization (Cheng and Lapata, 2016; Cao et al., 2017; Isonuma et al., 2017; Yasunaga et al., 2017; Narayan et al., 2018). These approaches remain extractive; and despite encouraging results, summarizing a large quantity of texts still requires sophisticated abstraction capabilities such as generalization, paraphrasing and sentence fusion.

Prior to deep learning, abstractive summarization has been investigated (Barzilay et al., 1999; Carenini and Cheung, 2008; Ganesan et al., 2010; Gerani et al., 2014; Fabbrizio et al., 2014; Pighin et al., 2014; Bing et al., 2015; Liu et al., 2015; Liao et al., 2018). These approaches construct domain templates using a text planner or an open-IE system and employ a natural language generator for surface realization. Limited by the availability of labelled data, experiments are often performed on small domain-specific datasets.

Neural abstractive summarization utilizing the encoder-decoder architecture has shown promising results but studies focus primarily on singledocument summarization (Nallapati et al., 2016; Kikuchi et al., 2016; Chen et al., 2016; Miao and Blunsom, 2016; Tan et al., 2017; Zeng et al., 2017; Zhou et al., 2017; Paulus et al., 2017; See et al., 2017; Gehrmann et al., 2018). The pointing mechanism (Gulcehre et al., 2016; Gu et al., 2016) allows a summarization system to both copy words from the source text and generate new words from the vocabulary. Reinforcement learning is exploited to directly optimize evaluation metrics (Paulus et al., 2017; Kryściński et al., 2018; Chen and Bansal, 2018). These studies fo- 
cus on summarizing single documents in part because the training data are abundant.

The work of Baumel et al. (2018) and Zhang et al. (2018) are related to ours. In particular, Baumel et al. (2018) propose to extend an abstractive summarization system to generate query-focused summaries; Zhang et al. (2018) add a document set encoder to their hierarchical summarization framework. With these few exceptions, little research has been dedicated to investigate the feasibility of extending the encoder-decoder framework to generate abstractive summaries from multi-document inputs, where available training data are scarce.

This paper presents some first steps towards the goal of extending the encoder-decoder model to a multi-document setting. We introduce an adaptation method combining the pointer-generator (PG) networks (See et al., 2017) and the maximal marginal relevance (MMR) algorithm (Carbonell and Goldstein, 1998). The PG model, trained on SDS data and detailed in Section $\S 3$, is capable of generating document abstracts by performing text abstraction and sentence fusion. However, if the model is applied at test time to summarize multi-document inputs, there will be limitations. Our PG-MMR algorithm, presented in Section $\S 4$, teaches the PG model to effectively recognize important content from the input documents, hence improving the quality of abstractive summaries, all without requiring any training on multidocument inputs.

\section{Limits of the Encoder-Decoder Model}

The encoder-decoder architecture has become the de facto standard for neural abstractive summarization (Rush et al., 2015). The encoder is often a bidirectional LSTM (Hochreiter and Schmidhuber, 1997) converting the input text to a set of hidden states $\left\{\mathbf{h}_{i}^{e}\right\}$, one for each input word, indexed by $i$. The decoder is a unidirectional LSTM that generates a summary by predicting one word at a time. The decoder hidden states are represented by $\left\{\mathbf{h}_{t}^{d}\right\}$, indexed by $t$. For sentence and singledocument summarization (Nallapati et al., 2016; Paulus et al., 2017; See et al., 2017), the input text is treated as a sequence of words, and the model is expected to capture the source syntax inherently.

$$
\begin{aligned}
e_{t, i} & =\mathbf{v}^{\top} \tanh \left(\mathbf{W}^{e}\left[\mathbf{h}_{t}^{d}\left\|\mathbf{h}_{i}^{e}\right\| \widetilde{\alpha}_{t, i}\right]+\mathbf{b}^{e}\right) \\
\alpha_{t, i} & =\operatorname{softmax}\left(e_{t, i}\right) \\
\widetilde{\alpha}_{t, i} & =\sum_{t^{\prime}=0}^{t-1} \alpha_{t^{\prime}, i}
\end{aligned}
$$

The attention weight $\alpha_{t, i}$ measures how impor- tant the $i$-th input word is to generating the $t$-th output word (Eq. (1-2)). Following (See et al., 2017), $\alpha_{t, i}$ is calculated by measuring the strength of interaction between the decoder hidden state $\mathbf{h}_{t}^{d}$, the encoder hidden state $\mathbf{h}_{i}^{e}$, and the cumulative attention $\widetilde{\alpha}_{t, i}$ (Eq. (3)). $\widetilde{\alpha}_{t, i}$ denotes the cumulative attention that the $i$-th input word receives up to time step $t-1$. A large value of $\widetilde{\alpha}_{t, i}$ indicates the $i$-th input word has been used prior to time $t$ and it is unlikely to be used again for generating the $t$-th output word.

A context vector $\left(\mathbf{c}_{t}\right)$ is constructed (Eq. (4)) to summarize the semantic meaning of the input; it is a weighted sum of the encoder hidden states. The context vector and the decoder hidden state $\left(\left[\mathbf{h}_{t}^{d} \| \mathbf{c}_{t}\right]\right)$ are then used to compute the vocabulary probability $P_{v c b}(w)$ measuring the likelihood of a vocabulary word $w$ being selected as the $t$-th output word (Eq. (5)). ${ }^{1}$

$$
\begin{aligned}
& \mathbf{c}_{t}=\sum_{i} \alpha_{t, i} \mathbf{h}_{i}^{e} \\
& P_{v c b}(w)=\operatorname{softmax}\left(\mathbf{W}^{y}\left[\mathbf{h}_{t}^{d} \| \mathbf{c}_{t}\right]+\mathbf{b}^{y}\right)
\end{aligned}
$$

In many encoder-decoder models, a "switch" is estimated ( $\left.p_{\text {gen }} \in[0,1]\right)$ to indicate whether the system has chosen to select a word from the vocabulary or to copy a word from the input text (Eq. (6)). The switch is computed using a feedforward layer with $\sigma$ activation over $\left[\mathbf{h}_{t}^{d}\left\|\mathbf{c}_{t}\right\| \mathbf{y}_{t-1}\right]$, where $\mathbf{y}_{t-1}$ is the embedding of the output word at time $t-1$. The attention weights $\left(\alpha_{t, i}\right)$ are used to compute the copy probability (Eq. (7)). If a word $w$ appears once or more in the input text, its copy probability $\left(\sum_{i: w_{i}=w} \alpha_{t, i}\right)$ is the sum of the attention weights over all its occurrences. The final probability $P(w)$ is a weighted combination of the vocabulary probability and the copy probability. A cross-entropy loss function can often be used to train the model end-to-end.

$$
\begin{aligned}
& \left.p_{\text {gen }}=\sigma\left(\mathbf{w}^{z}\left[\mathbf{h}_{t}^{d}\left\|\mathbf{c}_{t}\right\| \mathbf{y}_{t-1}\right]\right)+b^{z}\right) \\
& P(w)=p_{\text {gen }} P_{v c b}(w)+\left(1-p_{\text {gen }}\right) \sum_{i: w_{i}=w} \alpha_{t, i}
\end{aligned}
$$

To thoroughly understand the aforementioned encoder-decoder model, we divide its model parameters into four groups. They include

- parameters of the encoder and the decoder; - $\left\{\mathbf{w}^{z}, b^{z}\right\}$ for calculating the "switch" (Eq. (6));

\footnotetext{
${ }^{1}$ Here $[\cdot \| \cdot]$ represents the concatenation of two vectors. The pointer-generator networks (See et al., 2017) use two linear layers to produce the vocabulary distribution $P_{v c b}(w)$. We use $\mathbf{W}^{y}$ and $\mathbf{b}^{y}$ to denote parameters of both layers.
} 


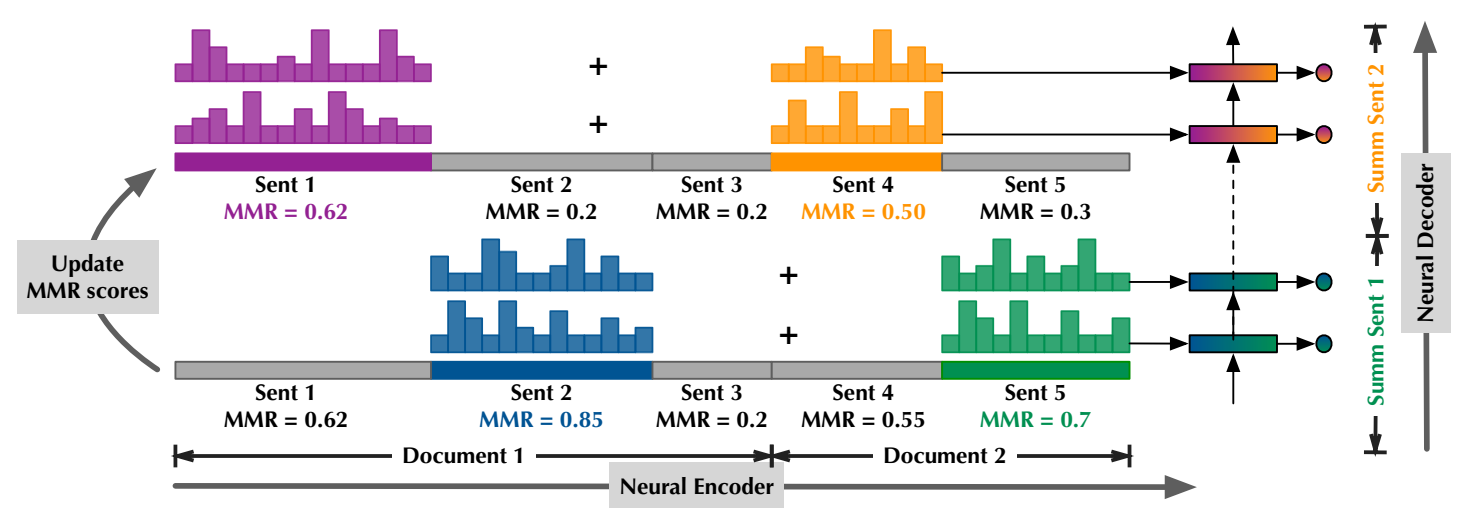

Figure 1: System framework. The PG-MMR system uses K highest-scored source sentences (in this case, K=2) to guide the PG model to generate a summary sentence. All other source sentences are "muted" in this process. Best viewed in color.

- $\left\{\mathbf{W}^{y}, \mathbf{b}^{y}\right\}$ for calculating $P_{v c b}(w)$ (Eq. (5));

- $\left\{\mathbf{v}, \mathbf{W}^{e}, \mathbf{b}^{e}\right\}$ for attention weights (Eq. (1)).

By training the encoder-decoder model on singledocument summarization (SDS) data containing a large collection of news articles paired with summaries (Hermann et al., 2015), these model parameters can be effectively learned.

However, at test time, we wish for the model to generate abstractive summaries from multidocument inputs. This brings up two issues. First, the parameters are ineffective at identifying salient content from multi-document inputs. Humans are very good at identifying representative sentences from a set of documents and fusing them into an abstract. However, this capability is not supported by the encoder-decoder model. Second, the attention mechanism is based on input word positions but not their semantics. It can lead to redundant content in the multi-document input being repeatedly used for summary generation. We conjecture that both aspects can be addressed by introducing an "external" model that selects representative sentences from multi-document inputs and dynamically adjusts the sentence importance to reduce summary redundancy. This external model is integrated with the encoder-decoder model to generate abstractive summaries using selected representative sentences. In the following section we present our adaptation method for multi-document summarization.

\section{Our Method}

Maximal marginal relevance. Our adaptation method incorporates the maximal marginal relevance algorithm (MMR; Carbonell and Goldstein, 1998) into pointer-generator networks (PG; See et al., 2017) by adjusting the network's attention values. MMR is one of the most successful extractive approaches and, despite its straightforwardness, performs on-par with state-of-the-art systems (Luo and Litman, 2015; Yogatama et al., 2015). At each iteration, MMR selects one sentence from the document $(D)$ and includes it in the summary $(S)$ until a length threshold is reached. The selected sentence $\left(s_{i}\right)$ is the most important one amongst the remaining sentences and it has the least content overlap with the current summary. In the equation below, $\operatorname{Sim}_{1}\left(s_{i}, D\right)$ measures the similarity of the sentence $s_{i}$ to the document. It serves as a proxy of sentence importance, since important sentences usually show similarity to the centroid of the document. $\max _{s_{j} \in S} \operatorname{Sim}_{2}\left(s_{i}, s_{j}\right)$ measures the maximum similarity of the sentence $s_{i}$ to each of the summary sentences, acting as a proxy of redundancy. $\lambda$ is a balancing factor.

$$
\underset{s_{i} \in D \backslash S}{\operatorname{argmax}}[\underbrace{\lambda \operatorname{Sim}_{1}\left(s_{i}, D\right)}_{\text {importance }}-\underbrace{(1-\lambda) \max _{s_{j} \in S} \operatorname{Sim}_{2}\left(s_{i}, s_{j}\right)}_{\text {redundancy }}]
$$

Our PG-MMR describes an iterative framework for summarizing a multi-document input to a summary consisting of multiple sentences. At each iteration, PG-MMR follows the MMR principle to select the K highest-scored source sentences; they serve as the basis for PG to generate a summary sentence. After that, the scores of all source sentences are updated based on their importance and redundancy. Sentences that are highly similar to the partial summary receive lower scores. Selecting $\mathrm{K}$ sentences via the MMR algorithm helps the PG system to effectively identify salient source content that has not been included in the summary.

Muting. To allow the PG system to effectively utilize the $\mathrm{K}$ source sentences without retraining the neural model, we dynamically adjust the PG attention weights $\left(\alpha_{t, i}\right)$ at test time. Let $\mathrm{S}_{k}$ rep- 
resent a selected sentence. The attention weights of the words belonging to $\left\{\mathbf{S}_{k}\right\}_{k=1}^{K}$ are calculated as before (Eq. (2)). However, words in other sentences are forced to receive zero attention weights $\left(\alpha_{t, i}=0\right)$, and all $\alpha_{t, i}$ are renormalized (Eq. (8)).

$$
\alpha_{t, i}^{\text {new }}= \begin{cases}\alpha_{t, i} & i \in\left\{\mathrm{S}_{k}\right\}_{k=1}^{K} \\ 0 & \text { otherwise }\end{cases}
$$

It means that the remaining sentences are "muted" in this process. In this variant, the sentence importance does not affect the original attention weights, other than muting.

In an alternative setting, the sentence salience is multiplied with the word salience and renormalized (Eq. (9)). PG uses the reweighted alpha values to predict the next summary word.

$$
\alpha_{t, i}^{\text {new }}= \begin{cases}\alpha_{t, i} \operatorname{MMR}\left(\mathbf{S}_{k}\right) & i \in\left\{\mathbf{S}_{k}\right\}_{k=1}^{K} \\ 0 & \text { otherwise }\end{cases}
$$

Sentence Importance. To estimate sentence importance $\operatorname{Sim}_{1}\left(s_{i}, D\right)$, we introduce a supervised regression model in this work. Importantly, the model is trained on single-document summarization datasets where training data are abundant. At test time, the model can be applied to identify important sentences from multi-document input. Our model determines sentence importance based on four indicators, inspired by how humans identify important sentences from a document set. They include (a) sentence length, (b) its absolute and relative position in the document, (c) sentence quality, and (d) how close the sentence is to the main topic of the document set. These features are considered to be important indicators in previous extractive summarization framework (Galanis and Androutsopoulos, 2010; Hong et al., 2014).

Regarding the sentence quality (c), we leverage the PG model to build the sentence representation. We use the bidirectional LSTM encoder to encode any source sentence to a vector representation. $\left[\overrightarrow{\mathbf{h}_{N}^{e}} \| \overleftarrow{\mathbf{h}_{1}^{e}}\right]$ is the concatenation of the last hidden states of the forward and backward passes. A document vector is the average of all sentence vectors. We use the document vector and the cosine similarity between the document and sentence vectors as indicator (d). A support vector regression model is trained on (sentence, score) pairs where the training data are obtained from the CNN/Daily Mail dataset. The target importance score is the ROUGE-L recall of the sentence compared to the ground-truth summary. Our model architecture leverages neural representations of sen-

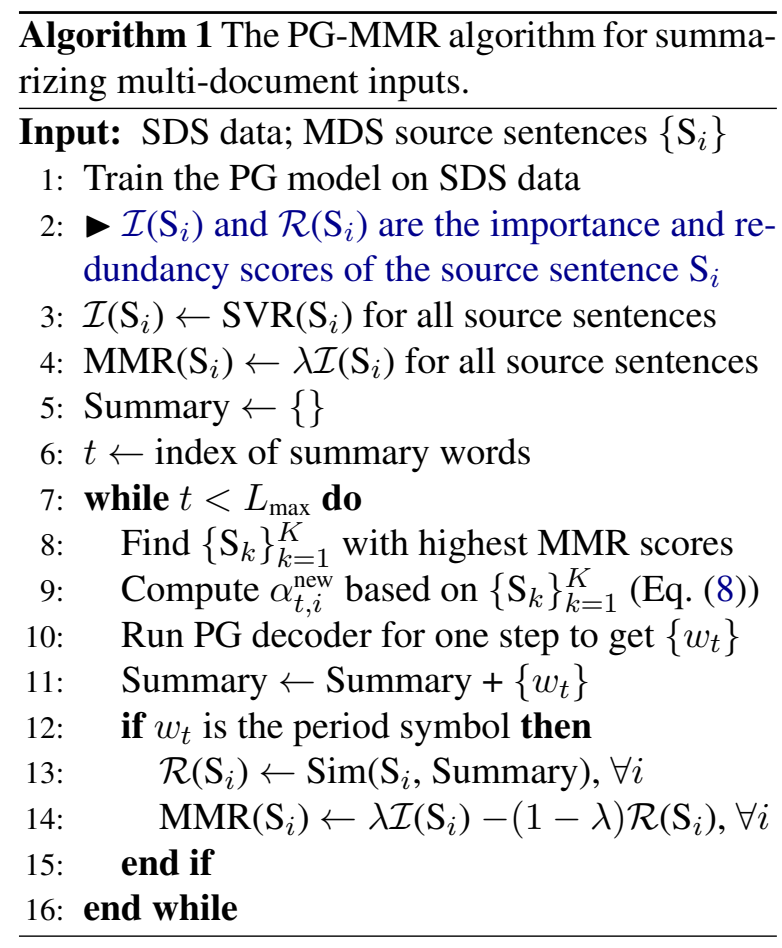

tences and documents, they are data-driven and not restricted to a particular domain.

Sentence Redundancy. To calculate the redundancy of the sentence $\left.\operatorname{(max}_{s_{j} \in S} \operatorname{Sim}_{2}\left(s_{i}, s_{j}\right)\right)$, we compute the ROUGE-L precision, which measures the longest common subsequence between a source sentence and the partial summary (consisting of all sentences generated thus far by the PG model), divided by the length of the source sentence. A source sentence yielding a high ROUGE$\mathrm{L}$ precision is deemed to have significant content overlap with the partial summary. It will receive a low MMR score and hence is less likely to serve as basis for generating future summary sentences.

Alg. 1 provides an overview the PG-MMR algorithm and Fig. 1 is a graphical illustration. The MMR scores of source sentences are updated after each summary sentence is generated by the PG model. Next, a different set of highest-scored sentences are used to guide the PG model to generate the next summary sentence. "Muting" the remaining source sentences is important because it helps the PG model to focus its attention on the most significant source content. The code for our model is publicly available to further MDS research. ${ }^{2}$

\section{Experimental Setup}

Datasets. We investigate the effectiveness of the PG-MMR method by testing it on standard multidocument summarization datasets (Over and Yen,

\footnotetext{
${ }^{2}$ https://github.com/ucfnlp/multidoc_summarization
} 
2004; Dang and Owczarzak, 2008). These include DUC-03, DUC-04, TAC-08, TAC-10, and TAC11 , containing 30/50/48/46/44 topics respectively. The summarization system is tasked with generating a concise, fluent summary of 100 words or less from a set of 10 documents discussing a topic. All documents in a set are chronologically ordered and concatenated to form a mega-document serving as input to the PG-MMR system. Sentences that start with a quotation mark or do not end with a period are excluded (Wong et al., 2008). Each system summary is compared against 4 human abstracts created by NIST assessors. Following convention, we report results on DUC-04 and TAC-11 datasets, which are standard test sets; DUC-03 and TAC-08/10 are used as a validation set for hyperparameter tuning. ${ }^{3}$

The PG model is trained for single-document summarization using the CNN/Daily Mail (Hermann et al., 2015) dataset, containing single news articles paired with summaries (human-written article highlights). The training set contains 287,226 articles. An article contains 781 tokens on average; and a summary contains 56 tokens (3.75 sentences). During training we use the hyperparameters provided by See et al. (2017). At test time, the maximum/minimum decoding steps are set to $120 / 100$ words respectively, corresponding to the $\mathrm{max} /$ min lengths of the PG-MMR summaries. Because the focus of this work is on multi-document summarization (MDS), we do not report results for the CNN/Daily Mail dataset.

Baselines. We compare PG-MMR against a broad spectrum of baselines, including state-of-the-art extractive ('ext-') and abstractive ('abs-') systems. They are described below. ${ }^{4}$

- ext-SumBasic (Vanderwende et al., 2007) is an extractive approach assuming words occurring frequently in a document set are more likely to be included in the summary;

- ext-KL-Sum (Haghighi and Vanderwende, 2009) greedily adds source sentences to the summary if it leads to a decrease in KL divergence;

- ext-LexRank (Erkan and Radev, 2004) uses a graph-based approach to compute sentence importance based on eigenvector centrality in a graph representation;

- ext-Centroid (Hong et al., 2014) computes the importance of each source sentence based on its cosine similarity with the document centroid;

- ext-ICSISumm (Gillick et al., 2009) leverages the ILP framework to identify a globally-optimal set of sentences covering the most important concepts in the document set;

\footnotetext{
${ }^{3}$ The hyperparameters for all PG-MMR variants are $K=7$ and $\lambda=0.6$; except for "w/ BestSummRec" where $K=2$.

${ }^{4} \mathrm{We}$ are grateful to Hong et al. (2014) for providing the summaries generated by Centroid, ICSISumm, DPP systems. These are only available for the DUC-04 dataset.
}

\begin{tabular}{|l|rrr|}
\hline & \multicolumn{3}{|c|}{ DUC-04 } \\
System & R-1 & R-2 & R-SU4 \\
\hline \hline SumBasic (Vanderwende et al., 2007) & 29.48 & 4.25 & 8.64 \\
KLSumm (Haghighi et al., 2009) & 31.04 & 6.03 & 10.23 \\
LexRank (Erkan and Radev, 2004) & 34.44 & 7.11 & 11.19 \\
Centroid (Hong et al., 2014) & 35.49 & 7.80 & 12.02 \\
ICSISumm (Gillick and Favre, 2009) & 37.31 & 9.36 & 13.12 \\
DPP (Taskar, 2012) & 38.78 & 9.47 & 13.36 \\
Extract+Rewrite (Song et al., 2018) & 28.90 & 5.33 & 8.76 \\
Opinosis (Ganesan et al., 2010) & 27.07 & 5.03 & 8.63 \\
PG-Original (See et al., 2017) & 31.43 & 6.03 & 10.01 \\
\hline PG-MMR w/ SummRec & 34.57 & 7.46 & 11.36 \\
PG-MMR w/ SentAttn & 36.52 & 8.52 & 12.57 \\
PG-MMR w/ Cosine (default) & 36.88 & $\mathbf{8 . 7 3}$ & 12.64 \\
\hline PG-MMR w/ BestSummRec & 36.42 & $\mathbf{9 . 3 6}$ & 13.23 \\
\hline
\end{tabular}

Table 2: ROUGE results on the DUC-04 dataset.

\begin{tabular}{|l|rrr|}
\hline & \multicolumn{3}{|c|}{ TAC-11 } \\
System & R-1 & R-2 & R-SU4 \\
\hline \hline SumBasic (Vanderwende et al., 2007) & 31.58 & 6.06 & 10.06 \\
KLSumm (Haghighi et al., 2009) & 31.23 & 7.07 & 10.56 \\
LexRank (Erkan and Radev, 2004) & 33.10 & 7.50 & 11.13 \\
Extract+Rewrite (Song et al., 2018) & 29.07 & 6.11 & 9.20 \\
Opinosis (Ganesan et al., 2010) & 25.15 & 5.12 & 8.12 \\
PG-Original (See et al., 2017) & 31.44 & 6.40 & 10.20 \\
\hline PG-MMR w/ SummRec & 35.06 & 8.72 & 12.39 \\
PG-MMR w/ SentAttn & 37.01 & 10.43 & 13.85 \\
PG-MMR w/ Cosine (default) & 37.17 & $\mathbf{1 0 . 9 2}$ & 14.04 \\
\hline PG-MMR w/ BestSummRec & 40.44 & $\mathbf{1 4 . 9 3}$ & 17.61 \\
\hline
\end{tabular}

Table 3: ROUGE results on the TAC-11 dataset.

- ext-DPP (Taskar, 2012) selects an optimal set of sentences per the determinantal point processes that balance the coverage of important information and the sentence diversity;

- abs-Opinosis (Ganesan et al., 2010) generates abstractive summaries by searching for salient paths on a word cooccurrence graph created from source documents;

- abs-Extract+Rewrite (Song et al., 2018) is a recent approach that scores sentences using LexRank and generates a title-like summary for each sentence using an encoderdecoder model trained on Gigaword data.

- abs-PG-Original (See et al., 2017) introduces an encoderdecoder model that encourages the system to copy words from the source text via pointing, while retaining the ability to produce novel words through the generator.

\section{Results}

Having described the experimental setup, we next compare the PG-MMR method against the baselines on standard MDS datasets, evaluated by both automatic metrics and human assessors.

ROUGE (Lin, 2004). This automatic metric measures the overlap of unigrams (R-1), bigrams (R2 ) and skip bigrams with a maximum distance of 4 words (R-SU4) between the system summary and a set of reference summaries. ROUGE scores of various systems are presented in Table 2 and 3 respectively for the DUC-04 and TAC-11 datasets.

We explore variants of the PG-MMR method. 
They differ in how the importances of source sentences are estimated and how the sentence importance affects word attention weights. "w/ Cosine" computes the sentence importance as the cosine similarity score between the sentence and document vectors, both represented as sparse TF-IDF vectors under the vector space model. "w/Summ$\boldsymbol{R e c}$ " estimates the sentence importance as the predicted R-L recall score between the sentence and the summary. A support vector regression model is trained on sentences from the CNN/Daily Mail datasets $(\approx 33 \mathrm{~K})$ and applied to DUC/TAC sentences at test time (see $\S 4$ ). "w/ BestSumm$\boldsymbol{R e c}$ " obtains the best estimate of sentence importance by calculating the R-L recall score between the sentence and reference summaries. It serves as an upper bound for the performance of " $\mathrm{w} /$ SummRec." For all variants, the sentence importance scores are normalized to the range of $[0,1]$. "w/ SentAttn" adjusts the attention weights using Eq. (9), so that words in important sentences are more likely to be used to generate the summary. The weights are otherwise computed using Eq. (8).

As seen in Table 2 and 3, our PG-MMR method surpasses all unsupervised extractive baselines, including SumBasic, KLSumm, and LexRank. On the DUC-04 dataset, ICSISumm and DPP show good performance, but these systems are trained directly on MDS datasets, which are not utilized by the PG-MMR method. PG-MMR exhibits superior performance compared to existing abstractive systems. It outperforms Opinosis and PGOriginal by a large margin in terms of R-2 F-scores (5.03/6.03/8.73 for DUC-04 and 5.12/6.40/10.92 for TAC-11). In particular, PG-Original is the original pointer-generator networks with multidocument inputs at test time. Compared to it, PGMMR is more effective at identifying summaryworthy content from the input. "w/ Cosine" is used as the default PG-MMR and it shows better results than "w/ SummRec." It suggests that the sentence and document representations obtained from the encoder-decoder model (trained on CNN/DM) are suboptimal, possibly due to a vocabulary mismatch, where certain words in the DUC/TAC datasets do not appear in CNN/DM and their embeddings are thus not learned during training. Finally, we observe that "w/ BestSummRec" yields the highest performance on both datasets. This finding suggests that there is a great potential for improvements of the PG-MMR method as its "extractive" and "abstractive" components can be separately optimized.

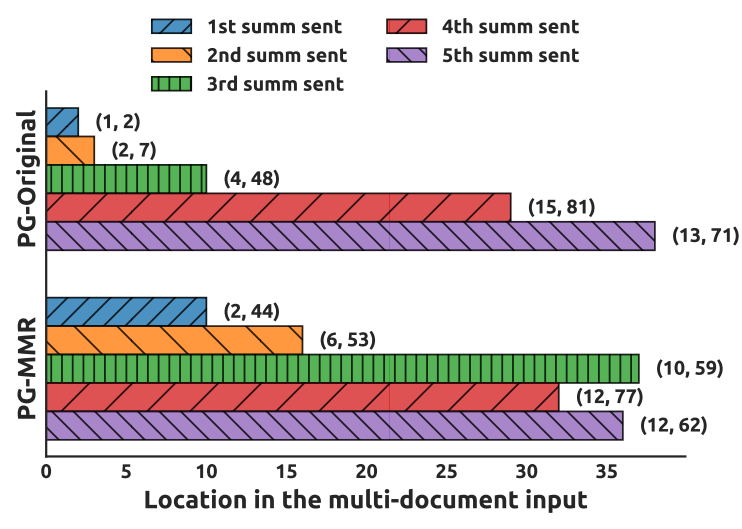

Figure 2: The median location of summary n-grams in the multi-document input (and the lower/higher quartiles). The n-grams come from the $1 \mathrm{st} / 2 \mathrm{nd} / 3 \mathrm{rd} / 4 \mathrm{th} / 5$ th summary sentence and the location is the source sentence index. (TAC-11)

\begin{tabular}{|l|cccc|}
\hline System & 1-grams & 2-grams & 3-grams & Sent \\
\hline \hline Extr+Rewrite & 89.37 & 54.34 & 25.10 & 6.65 \\
PG-Original & 99.64 & 96.28 & 88.83 & 47.67 \\
PG-MMR & 99.74 & 97.64 & 91.57 & 59.13 \\
Human Abst. & 84.32 & 45.22 & 18.70 & 0.23 \\
\hline
\end{tabular}

Table 4: Percentages of summary n-grams (or the entire sentences) appear in the multi-document input. (TAC-11)

Location of summary content. We are interested in understanding why PG-MMR outperforms PG-Original at identifying summary content from the multi-document input. We ask the question: where, in the source documents, does each system tend to look when generating their summaries? Our findings indicate that PG-Original gravitates towards early source sentences, while PG-MMR searches beyond the first few sentences.

In Figure 2 we show the median location of the first occurrences of summary n-grams, where the n-grams can come from the 1st to 5th summary sentence. For PG-Original summaries, n-grams of the 1st summary sentence frequently come from the 1st and 2nd source sentences, corresponding to the lower/higher quartiles of source sentence indices. Similarly, n-grams of the 2nd summary sentence come from the 2 nd to 7 th source sentences. For PG-MMR summaries, the patterns are different. The n-grams of the 1 st and 2nd summary sentences come from source sentences of the range $(2,44)$ and $(6,53)$, respectively. Our findings suggest that PG-Original tends to treat the input as a single-document and identifies summary-worthy content from the beginning of the input, whereas PG-MMR can successfuly search a broader range of the input for summary content. This capability is crucial for multi-document input where important content can come from any article in the set.

Degree of extractiveness. Table 4 shows the 


\begin{tabular}{|l|ccc|rcrr|}
\hline & \multicolumn{3}{|c|}{ Linguistic Quality } & \multicolumn{4}{c|}{ Rankings (\%) } \\
System & Fluency & Inform. & NonRed. & 1st & 2nd & 3rd & 4th \\
\hline \hline Extract+Rewrite & 2.03 & 2.19 & 1.88 & 5.6 & 11.6 & 11.6 & $\mathbf{7 1 . 2}$ \\
LexRank & $\mathbf{3 . 2 9}$ & 3.36 & 3.30 & 30.0 & 28.8 & 32.0 & 9.2 \\
PG-Original & 3.20 & 3.30 & 3.19 & 29.6 & 26.8 & $\mathbf{3 2 . 8}$ & 10.8 \\
PG-MMR & 3.24 & $\mathbf{3 . 5 2}$ & $\mathbf{3 . 4 2}$ & $\mathbf{3 4 . 8}$ & $\mathbf{3 2 . 8}$ & 23.6 & 8.8 \\
\hline
\end{tabular}

Table 5: Linguistic quality and rankings of system summaries. (DUC-04)

\begin{tabular}{l} 
Human Abstract \\
- Boeing 737-400 plane with 102 people on board crashed into a moun- \\
tain in the West Sulawesi province of Indonesia, on Monday, January \\
01,2007, killing at least 90 passengers, with 12 possible survivors. \\
- The plane was Adam Air flight KI-574, departing at $12: 59$ pm from \\
Surabaya on Java bound for Manado in northeast Sulawesi. \\
- The plane crashed in a mountainous region in Polewali, west Su- \\
lawesi province. \\
- There were three Americans on board, it is not know if they \\
survived. \\
- The cause of the crash is not known at this time but it is possible bad \\
weather was a factor. \\
\hline Extract+Rewrite Summary \\
- Plane with 102 people on board crashes. \\
- Three Americans among 102 on board plane in Indonesia. \\
- Rescue team arrives in Indonesia after plane crash. \\
- Plane with 102 crashes in West Sulawesi, killing at least 90. \\
- No word on the fate of Boeing 737-400. \\
- Plane carrying 96 passengers loses contact with Makassar. \\
- Plane crashes in Indonesia, killing at least 90. \\
- Indonesian navy sends two planes to carry bodies of five. \\
- Indonesian plane carrying 102 missing. \\
- Indonesian lawmaker criticises slow deployment of plane. \\
- Hundreds of kilometers plane crash.
\end{tabular}

\begin{tabular}{l} 
PG-Original Summary \\
- Adam Air Boeing $737-400$ crashed Monday after vanishing off air \\
traffic control radar screens between the Indonesian islands of Java and \\
Sulawesi. \\
- Up to 12 people were thought to have survived, with rescue teams \\
racing to the crash site near Polewali in West Sulawesi, some 180 kilo- \\
metres north of the South Sulawesi provincial capital Makassar. \\
- It was the worst air disaster since Sept. 5, 2005, when a Mandala Air- \\
line's Boeing $737-200$ crashed shortly after taking off from the North \\
Sumatra's airport, killing 103 people. \\
- Earlier on Friday, a ferry carrying 628 people sank off the Java coast. \\
\hline PG-MMR Summary \\
- The Adam Air Boeing $737-400$ crashed Monday afternoon, but \\
search and rescue teams only discovered the wreckage early Tuesday. \\
- The Indonesian rescue team arrived at the mountainous area in West \\
Sulawesi province where a passenger plane with 102 people onboard \\
crashed into a mountain in Polewali, West Sulawesi province. \\
- Air force rear commander Eddy Suyanto told-Shinta radio station \\
that the plane - operated by local carrier Adam Air - had crashed in a \\
mountainous region in Polewali province on Monday. \\
- There was no word on the fate of the remaining 12 people on board \\
the boeing 737-400.
\end{tabular}

Table 6: Example system summaries and human-written abstract. The sentences are manually de-tokenized for readability.

percentages of summary n-grams (or entire sentences) appearing in the multi-document input. PG-Original and PG-MMR summaries both show a high degree of extractiveness, and similar findings have been revealed by See et al. (2017). Because PG-MMR relies on a handful of representative source sentences and mutes the rest, it appears to be marginally more extractive than PG-Original. Both systems encourage generating summary sentences by stitching together source sentences, as about $52 \%$ and $41 \%$ of the summary sentences do not appear in the source, but about $90 \%$ the n-grams do. The Extract+Rewrite summaries ( $(5)$, generated by rewriting selected source sentences to title-like summary sentences, exhibits a high degree of abstraction, close to that of human abstracts.

Linguistic quality. To assess the linguistic quality of various system summaries, we employ Amazon Mechanical Turk human evaluators to judge the summary quality, including PG-MMR, LexRank, PG-Original, and Extract+Rewrite. A turker is asked to rate each system summary on a scale of 1 (worst) to 5 (best) based on three evaluation criteria: informativeness (to what extent is the meaning expressed in the ground-truth text preserved in the summary?), fluency (is the summary grammatical and well-formed?), and non-redundancy (does the summary successfully avoid repeating information?). Human summaries are used as the ground-truth. The turkers are also asked to provide an overall ranking for the four system summaries. Results are presented in Table 5. We observe that the LexRank summaries are highest-rated on fluency. This is because LexRank is an extractive approach, where summary sentences are directly taken from the input. PG-MMR is rated as the best on both informativeness and non-redundancy. Regarding overall system rankings, PG-MMR summaries are frequently ranked as the 1st- and 2 ndbest summaries, outperforming the others.

Example summaries. In Table 6 we present example summaries generated by various systems. PG-Original cannot effectively identify important content from the multi-document input. Extract+Rewrite tends to generate short, title-like sentences that are less informative and carry substantial redundancy. This is because the system is trained on the Gigaword dataset (Rush et al., 2015) where the target summary length is 7 words. PGMMR generates summaries that effectively condense the important source content. 


\section{Conclusion}

We describe a novel adaptation method to generate abstractive summaries from multi-document inputs. Our method combines an extractive summarization algorithm (MMR) for sentence extraction and a recent abstractive model (PG) for fusing source sentences. The PG-MMR system demonstrates competitive results, outperforming strong extractive and abstractive baselines.

\section{References}

Dzmitry Bahdanau, Kyunghyun Cho, and Yoshua Bengio. 2014. Neural machine translation by jointly learning to align and translate. CoRR, abs/1409.0473.

Regina Barzilay, Kathleen R. McKeown, and Michael Elhadad. 1999. Information fusion in the context of multi-document summarization. In Proceedings of the Annual Meeting of the Association for Computational Linguistics (ACL).

Tal Baumel, Matan Eyal, and Michael Elhadad. 2018. Query focused abstractive summarization: Incorporating query relevance, multi-document coverage, and summary length constraints into seq2seq models. arXiv preprint arXiv:1801.07704.

Taylor Berg-Kirkpatrick, Dan Gillick, and Dan Klein. 2011. Jointly learning to extract and compress. In Proceedings of the Annual Meeting of the Association for Computational Linguistics (ACL).

Lidong Bing, Piji Li, Yi Liao, Wai Lam, Weiwei Guo, and Rebecca J. Passonneau. 2015. Abstractive multi-document summarization via phrase selection and merging. In Proceedings of ACL.

Ziqiang Cao, Wenjie Li, Sujian Li, and Furu Wei. 2017. Improving multi-document summarization via text classification. In Proceedings of the Association for the Advancement of Artificial Intelligence (AAAI).

Jaime Carbonell and Jade Goldstein. 1998. The use of MMR, diversity-based reranking for reordering documents and producing summaries. In Proceedings of the International ACM SIGIR Conference on Research and Development in Information Retrieval (SIGIR).

Giuseppe Carenini and Jackie Chi Kit Cheung. 2008. Extractive vs. NLG-based abstractive summarization of evaluative text: The effect of corpus controversiality. In Proceedings of the Fifth International Natural Language Generation Conference (INLG).

Qian Chen, Xiaodan Zhu, Zhen-Hua Ling, Si Wei, and Hui Jiang. 2016. Distraction-based neural networks for document summarization. In Proceedings of the Twenty-Fifth International Joint Conference on Artificial Intelligence (IJCAI).
Yen-Chun Chen and Mohit Bansal. 2018. Fast abstractive summarization with reinforce-selected sentence rewriting. In Proceedings of the Annual Meeting of the Association for Computational Linguistics (ACL).

Jianpeng Cheng and Mirella Lapata. 2016. Neural summarization by extracting sentences and words. In Proceedings of ACL.

Hoa Trang Dang and Karolina Owczarzak. 2008. Overview of the TAC 2008 update summarization task. In Proceedings of Text Analysis Conference (TAC).

Hal Daume III and Daniel Marcu. 2002. A noisychannel model for document compression. In Proceedings of the Annual Meeting of the Association for Computational Linguistics (ACL).

Greg Durrett, Taylor Berg-Kirkpatrick, and Dan Klein. 2016. Learning-based single-document summarization with compression and anaphoricity constraints. In Proceedings of the Association for Computational Linguistics (ACL).

Günes Erkan and Dragomir R. Radev. 2004. LexRank: Graph-based lexical centrality as salience in text summarization. Journal of Artificial Intelligence Research.

Giuseppe Di Fabbrizio, Amanda J. Stent, and Robert Gaizauskas. 2014. A hybrid approach to multidocument summarization of opinions in reviews. Proceedings of the 8th International Natural Language Generation Conference (INLG).

Katja Filippova, Enrique Alfonseca, Carlos Colmenares, Lukasz Kaiser, and Oriol Vinyals. 2015. Sentence compression by deletion with lstms. In Proceedings of the Conference on Empirical Methods in Natural Language Processing (EMNLP).

Dimitrios Galanis and Ion Androutsopoulos. 2010. An extractive supervised two-stage method for sentence compression. In Proceedings of NAACL-HLT.

Kavita Ganesan, ChengXiang Zhai, and Jiawei Han. 2010. Opinosis: A graph-based approach to abstractive summarization of highly redundant opinions. In Proceedings of the International Conference on Computational Linguistics (COLING).

Sebastian Gehrmann, Yuntian Deng, and Alexander Rush. 2018. Bottom-up abstractive summarization. In Proceedings of the Conference on Empirical Methods in Natural Language Processing (EMNLP).

Shima Gerani, Yashar Mehdad, Giuseppe Carenini, Raymond T. Ng, and Bita Nejat. 2014. Abstractive summarization of product reviews using discourse structure. In Proceedings of the Conference on Empirical Methods in Natural Language Processing (EMNLP). 
Dan Gillick and Benoit Favre. 2009. A scalable global model for summarization. In Proceedings of the NAACL Workshop on Integer Linear Programming for Natural Langauge Processing.

Dan Gillick, Benoit Favre, Dilek Hakkani-Tur, Berndt Bohnet, Yang Liu, and Shasha Xie. 2009. The ICSI/UTD summarization system at TAC 2009. In Proceedings of TAC.

Max Grusky, Mor Naaman, and Yoav Artzi. 2018. Newsroom: A dataset of 1.3 million summaries with diverse extractive strategies. In Proceedings of the North American Chapter of the Association for Computational Linguistics (NAACL).

Jiatao Gu, Zhengdong Lu, Hang Li, and Victor O.K. Li. 2016. Incorporating copying mechanism in sequence-to-sequence learning. In Proceedings of $A C L$.

Caglar Gulcehre, Sungjin Ahn, Ramesh Nallapati, Bowen Zhou, and Yoshua Bengio. 2016. Pointing the unknown words. In Proceedings of the 54th Annual Meeting of the Association for Computational Linguistics (ACL).

Aria Haghighi and Lucy Vanderwende. 2009. Exploring content models for multi-document summarization. In Proceedings of the North American Chapter of the Association for Computational Linguistics (NAACL).

Karl Moritz Hermann, Tomas Kocisky, Edward Grefenstette, Lasse Espeholt, Will Kay, Mustafa Suleyman, and Phil Blunsom. 2015. Teaching machines to read and comprehend. In Proceedings of Neural Information Processing Systems (NIPS).

Sepp Hochreiter and Jurgen Schmidhuber. 1997. Long short-term memory. Neural Computation, 9(8):1735-1780.

Kai Hong, John M Conroy, Benoit Favre, Alex Kulesza, Hui Lin, and Ani Nenkova. 2014. A repository of state of the art and competitive baseline summaries for generic news summarization. In Proceedings of the Ninth International Conference on Language Resources and Evaluation (LREC).

Masaru Isonuma, Toru Fujino, Junichiro Mori, Yutaka Matsuo, and Ichiro Sakata. 2017. Extractive summarization using multi-task learning with document classification. In Proceedings of the Conference on Empirical Methods in Natural Language Processing (EMNLP).

Hongyan Jing and Kathleen McKeown. 1999. The decomposition of human-written summary sentences. In Proceedings of the International ACM SIGIR Conference on Research and Development in Information Retrieval (SIGIR).

Yuta Kikuchi, Graham Neubig, Ryohei Sasano, Hiroya Takamura, and Manabu Okumura. 2016. Controlling output length in neural encoder-decoders. In Proceedings of EMNLP.
Wojciech Kryściński, Romain Paulus, Caiming Xiong, and Richard Socher. 2018. Improving abstraction in text summarization. In Proceedings of the Conference on Empirical Methods in Natural Language Processing (EMNLP).

Chen Li, Fei Liu, Fuliang Weng, and Yang Liu. 2013. Document summarization via guided sentence compression. In Proceedings of the 2013 Conference on Empirical Methods in Natural Language Processing (EMNLP).

Kexin Liao, Logan Lebanoff, and Fei Liu. 2018. Abstract meaning representation for multi-document summarization. In Proceedings of the International Conference on Computational Linguistics (COL$I N G)$.

Chin-Yew Lin. 2004. ROUGE: a package for automatic evaluation of summaries. In Proceedings of ACL Workshop on Text Summarization Branches Out.

Fei Liu, Jeffrey Flanigan, Sam Thomson, Norman Sadeh, and Noah A. Smith. 2015. Toward abstractive summarization using semantic representations. In Proceedings of the North American Chapter of the Association for Computational Linguistics: $\mathrm{Hu}$ man Language Technologies (NAACL).

Wencan Luo and Diane Litman. 2015. Summarizing student responses to reflection prompts. In Proceedings of the Conference on Empirical Methods in Natural Language Processing (EMNLP).

Wencan Luo, Fei Liu, Zitao Liu, and Diane Litman. 2016. Automatic summarization of student course feedback. In Proceedings of the North American Chapter of the Association for Computational Linguistics: Human Language Technologies (NAACL).

Yishu Miao and Phil Blunsom. 2016. Language as a latent variable: Discrete generative models for sentence compression. In Proceedings of the Conference on Empirical Methods in Natural Language Processing (EMNLP).

Ramesh Nallapati, Bowen Zhou, Cicero dos Santos, Caglar Gulcehre, and Bing Xiang. 2016. Abstractive text summarization using sequence-tosequence RNNs and beyond. In Proceedings of the 20th SIGNLL Conference on Computational Natural Language Learning (CoNLL).

Shashi Narayan, Shay B. Cohen, and Mirella Lapata. 2018. Ranking sentences for extractive summarization with reinforcement learning. In Proceedings of the 16th Annual Conference of the North American Chapter of the Association for Computational Linguistics: Human Language Technologies (NAACL$H L T)$.

Ani Nenkova and Kathleen McKeown. 2011. Automatic summarization. Foundations and Trends in Information Retrieval. 
Paul Over and James Yen. 2004. An introduction to DUC-2004. National Institute of Standards and Technology.

Romain Paulus, Caiming Xiong, and Richard Socher. 2017. A deep reinforced model for abstractive summarization. In Proceedings of the Conference on Empirical Methods in Natural Language Processing (EMNLP).

Daniele Pighin, Marco Cornolti, Enrique Alfonseca, and Katja Filippova. 2014. Modelling events through memory-based, open-ie patterns for abstractive summarization. In Proceedings of the Annual Meeting of the Association for Computational Linguistics (ACL).

Alexander M. Rush, Sumit Chopra, and Jason Weston. 2015. A neural attention model for sentence summarization. In Proceedings of the Conference on Empirical Methods in Natural Language Processing (EMNLP).

Evan Sandhaus. 2008. The new york times annotated corpus. Linguistic Data Consortium.

Abigail See, Peter J. Liu, and Christopher D. Manning. 2017. Get to the point: Summarization with pointergenerator networks. In Proceedings of the Annual Meeting of the Association for Computational Linguistics (ACL).

Kaiqiang Song, Lin Zhao, and Fei Liu. 2018. Structure-infused copy mechanisms for abstractive summarization. In Proceedings of the International Conference on Computational Linguistics (COLING).

Sho Takase, Jun Suzuki, Naoaki Okazaki, Tsutomu Hirao, and Masaaki Nagata. 2016. Neural headline generation on abstract meaning representation. In Proceedings of the Conference on Empirical Methods in Natural Language Processing (EMNLP).

Jiwei Tan, Xiaojun Wan, and Jianguo Xiao. 2017. Abstractive document summarization with a graphbased attentional neural model. In Proceedings of the Annual Meeting of the Association for Computational Linguistics (ACL).

Alex Kuleszaand Ben Taskar. 2012. Determinantal Point Processes for Machine Learning. Now Publishers Inc.

Kapil Thadani and Kathleen McKeown. 2013. Sentence compression with joint structural inference. In Proceedings of CoNLL.

Lucy Vanderwende, Hisami Suzuki, Chris Brockett, and Ani Nenkova. 2007. Beyond SumBasic: Taskfocused summarization with sentence simplification and lexical expansion. Information Processing and Management, 43(6):1606-1618.
Lu Wang, Hema Raghavan, Vittorio Castelli, Radu Florian, and Claire Cardie. 2013. A sentence compression based framework to query-focused multidocument summarization. In Proceedings of ACL.

Kam-Fai Wong, Mingli Wu, and Wenjie Li. 2008. Extractive summarization using supervised and semisupervised learning. In Proceedings of the International Conference on Computational Linguistics (COLING).

Michihiro Yasunaga, Rui Zhang, Kshitijh Meelu, Ayush Pareek, Krishnan Srinivasan, and Dragomir Radev. 2017. Graph-based neural multi-document summarization. In Proceedings of the Conference on Computational Natural Language Learning (CoNLL).

Dani Yogatama, Fei Liu, and Noah A. Smith. 2015. Extractive summarization by maximizing semantic volume. In Proceedings of the Conference on Empirical Methods on Natural Language Processing (EMNLP).

David Zajic, Bonnie J. Dorr, Jimmy Lin, and Richard Schwartz. 2007. Multi-candidate reduction: Sentence compression as a tool for document summarization tasks. Information Processing and Management.

Wenyuan Zeng, Wenjie Luo, Sanja Fidler, and Raquel Urtasun. 2017. Efficient summarization with readagain and copy mechanism. In Proceedings of the International Conference on Learning Representations (ICLR).

Jianmin Zhang, Jiwei Tan, and Xiaojun Wan. 2018. Towards a neural network approach to abstractive multi-document summarization. arXiv preprint arXiv:1804.09010.

Qingyu Zhou, Nan Yang, Furu Wei, and Ming Zhou. 2017. Selective encoding for abstractive sentence summarization. In Proceedings of the Annual Meeting of the Association for Computational Linguistics (ACL). 